County, plants sprayed with stylet oil had about $10.4 \%$ virus infection, compared to about $13.3 \%$ for those not sprayed. Virus incidence in the two treatments was not statistically different $(P=0.05)$.

\section{Conclusion}

Virus spread was extremely rapid in the first two trials at Davis. Virus incidence increased from almost zero to about $35 \%$ in the first trial, and to about $80 \%$ in the second trial, just one week after symptoms were initially noticed.

Our observations and experiments indicate that low numbers of aphids are capable of causing a high level of disease if a source of virus inoculum is nearby. Viruses such as WMV and CMV are spread by non-colonizing aphids, so growers can't rely on visible evidence of aphids colonizing melon plants as an indication of infection.

Based on the results of our experiments, we concluded that JMS Stylet Oil is effective in reducing the incidence and spread of aphid-transmitted viruses when inoculum pressure is low. When inoculum pressure is high, the stylet oil appears to delay the initial infection by a few days but does not succeed in keeping the spread at tolerable levels. Researchers in Florida recently reported similar results.

Given these limitations, growers must decide for themselves whether application of JMS Stylet Oil is costeffective. The application of stylet oil requires a significant amount of labor and cost. Thorough coverage of plants is necessary at all times to prevent aphid virus transmission. This requirement may be difficult to meet given the rapid growth of melon plants during warm growing seasons.

K.C. Umesh is Postdoctoral Researcher, C. Hurley is Research Assistant, W.D. Gubler is Professor and Extension Pathologist, and B.W. Falk is Professor, all in the Department of Plant Pathology, UC Davis; and J. Valencia is Farm Advisor, UC Cooperative Extension, Stanislaus County.

We thank the California Melon Research Board for funding part of this study.

\title{
Efforts to reduce stratospheric ozone loss affect agriculture
}

\author{
Bryan C. Weare
}

\begin{abstract}
Research has shown that the increased ultraviolet radiation reaching the Earth's surface resulting from stratospheric ozone loss poses a danger to everyone. Concern about ozone loss prompted many nations to ratify the Montreal Protocol, the most comprehensive international environmental agreement ever enacted. Several provisions of this protocol will have substantial, long-term effects on the agricultural industry. Agriculture contributes substantially to ozone depletion, primarily through its use of chlorofluorocarbons (CFCs) for refrigeration in processing, storage and transport of meats and produce. This paper is meant to serve as an overview of the scientific basis for ozone depletion concerns, a description of the current international policy agreement, and the possible consequences of that policy for agriculture.
\end{abstract}

Ozone is a rare form of oxygen that is composed of three $\left(\mathrm{O}_{3}\right)$ rather than two atoms $\left(\mathrm{O}_{2}\right)$ as is true of the vast majority of oxygen in the atmosphere. Ozone exists primarily in two distinct layers of the atmosphere: in the bottom mile, especially in urban regions, and 15 to 30 miles above ground in the stratosphere. Ozone in the lower level is the most important form of urban air pollution and might be labeled "bad" ozone because it contributes to respiratory and other health problems and increased concentrations may lead to decreased crop yields. The second layer is much thicker and is essential to all life as the primary absorber of dangerous ultraviolet radiation from the sun's rays. This might be called the "good" ozone. This report will only be concerned with this stratospheric ozone layer.

\section{Stratospheric ozone depletion}

The basics of the science of stratospheric ozone and its depletion by chlorofluorocarbons (CFCs) are illustrated schematically in figure 1 . Good ozone is continually created and destroyed by a number of complex chemical reactions. The ultimate source of the ozone is the simple reaction $\mathrm{O}_{2}+\mathrm{O}=\mathrm{O}_{3}$; the oxygen atom $\mathrm{O}$ is formed when the sun's ultraviolet radiation breaks apart an oxygen molecule $\mathrm{O}_{2}$. The ultimate loss of ozone is through nearly the opposite reaction $\mathrm{O}_{3}+\mathrm{O}=2 \mathrm{O}_{2}$ that results in regular oxygen molecules again. The actual processes are considerably more complicated, involving catalysts which help speed production or destruction. Without human intervention, the production and destruction processes result in a relatively fixed amount of ozone, much like a bathtub would remain at a constant level if the amount of water flowing from the tap exactly equaled the amount going down the drain .

Human intervention does occur and affects the amount of ozone in the stratosphere. Ozone depletion is primarily due to the introduction of large quantities of chlorine- and brominecontaining molecules into the stratosphere. The most important are re- 
leased from chlorofluorocarbon (CFC) molecules as they are broken down by the sun's ultraviolet radiation. These CFCs, usually referred to by their brand name Freon, were developed in the 1930s as the "perfect" refrigerant because they are efficient, non-toxic and non-reactive. They are also used extensively as foam-blowing agents and cleaning agents in the computer and other industries. The most common are often referred to by abbreviations CFC-11 and CFC-12.

Early in the 1970s, scientists realized that CFCs move slowly into the stratosphere, becoming reactive in the presence of ultraviolet sunlight. Ultimately these CFCs break down into their various components. Some resulting chlorine-containing molecules can act as powerful catalysts, greatly speeding the destruction of ozone and reducing the overall concentration of stratospheric ozone. Furthermore, as with all catalysts, the chlorine atoms are usually unharmed by these reactions. In fact, one chlorine molecule can destroy thousands of molecules of ozone.

The details are complex. For instance, the chlorine is much more effective at destroying ozone in the presence of small particles. Such particles are rare in the stratosphere. They occur in large numbers only after a strong volcanic eruption, or in polar regions in winter and spring, when the air is cold enough for clouds to form even in the very dry stratosphere. This is the reason for the most dramatic manifestation of ozone depletion, the so-called "Antarctic ozone hole." This hole - really a dramatic decrease in ozone - develops in the spring when the air is cold, stratospheric clouds are prevalent and the necessary sunlight is present, enabling the chemical reactions to occur. Fortunately, this hole is most evident for only about a month. Ozone concentrations return to near normal when spring warms the Southern Hemisphere and the stratospheric clouds disappear.

In the past 10 years, this theory has been well verified and corroborated through observations of the stratosphere. The most dramatic observation has been the steady, sharp decline in

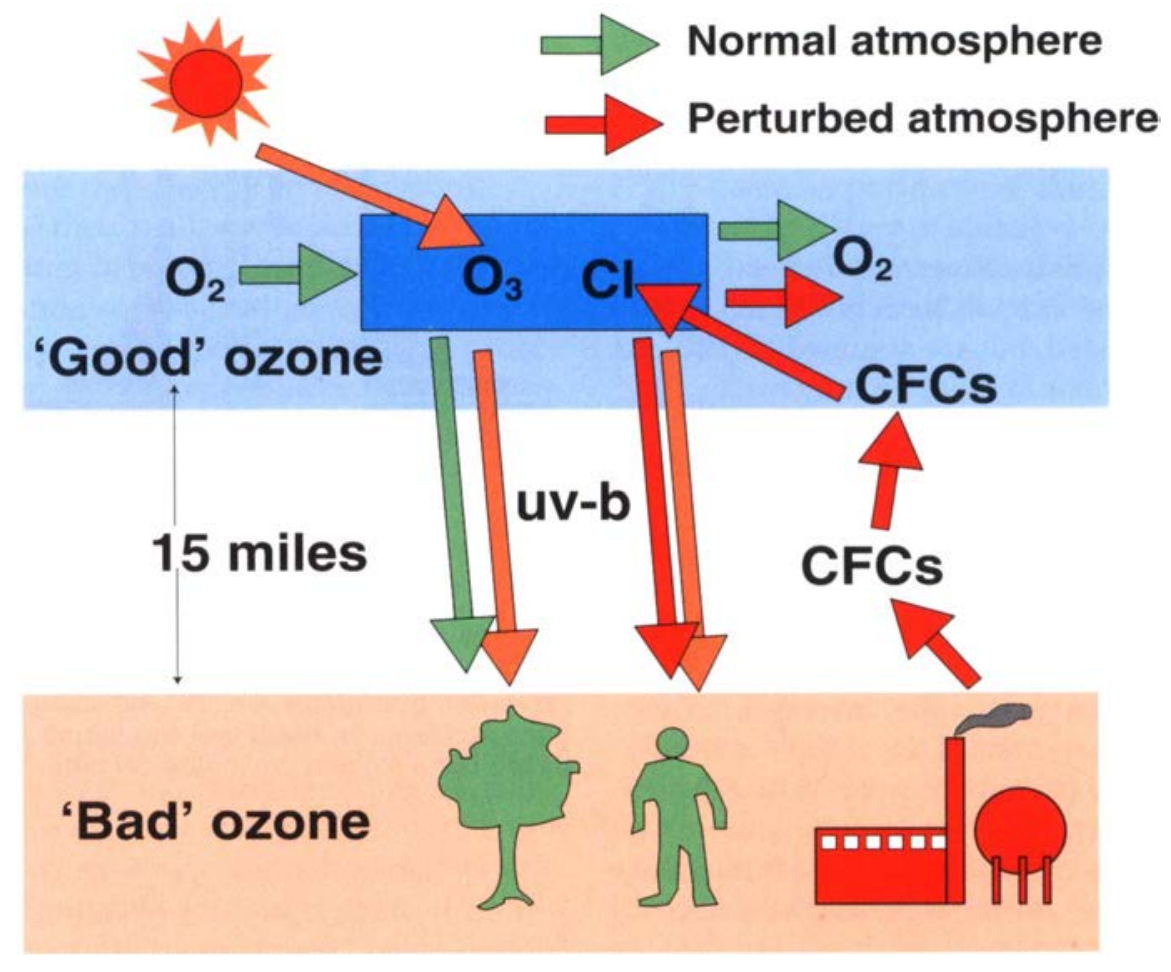

Fig. 1. Schematic of atmospheric ozone and the effects of CFCs as explained in the text.

measured Antarctic ozone every October for the past 15 years. Ozone concentrations over Antarctica have been at record lows for each successive year beginning in October 1992. Using high-flying aircraft and satellites, scientists have found not only decreased ozone levels associated with the hole, but also increased concentrations of the chlorine-containing molecules that destroy ozone in the laboratory. Few scientists now dispute that ozone depletion over the Antarctic is occurring and that it is due to CFCs and related anthropogenic chemicals released into the atmosphere.

The Antarctic ozone hole is only the clearest manifestation of decreases in stratospheric ozone concentrations. Ozone destruction is occurring worldwide, albeit at slower rates elsewhere. Other losses are due in part to the residual effect of the Antarctic hole, much in the way red paint (air depleted of ozone) mixes with white paint (air rich in ozone) to create a pale pink (air with decreased ozone concentrations). Recent measurements show that a 2 to $20 \%$ decrease in ozone concentration occurred in all parts of the world between 1979 and 1992 (fig. 2A). Although these decreases are largest in the polar regions, reductions of about $7 \%$ exist in the heavily populated regions of the Northern Hemisphere between $35^{\circ}$ and $65^{\circ} \mathrm{N}$.

The primary importance of this substantial ozone depletion is in the resulting increase of destructive ultraviolet radiation, especially so-called $u v-b$, reaching the ground. There is reliable evidence that increased exposure to uv-b can lead to significant increases in skin cancers (including nearly always fatal melanomas), cataracts and immune deficiencies in both humans and animals, as well as the destruction of genetic material in plants of all kinds. Figure 2B shows a calculation of the increase in a skin carcinoma between 1979 and 1992, utilizing the measured ozone decreases shown in figure $2 \mathrm{~A}$ and laboratory estimates of how increased uv-b damages cells. Again, the largest percentage increases in carcinomas are in polar regions where few people live and even fewer people spend hours outside sunbathing. However, significant increases are predicted in the heavily populated regions of the Northern Hemisphere, where increases in surface uv-b due to losses in stratospheric ozone may be offset somewhat by 
increases in the "bad" surface ozone associated with air pollution. Unfortunately, it will probably be years before longterm studies of cancer patients will be available to verify these predicted trends. The effects on animals have not been estimated, but are assumed to be similar to those on humans.

The overall influence of modest increases in uv-b radiation on plants is not well understood. Nevertheless, there is important evidence suggesting that increases of uv-b can lead to decreased crop yields. For instance, one study suggested that increases in $u v-b$ of $20 \%$ decreased the yield of a sensitive soybean cultivar by 19 to $25 \%$ in 5 out of 6 years. In other cases, more uv-b was found to increase branching, and shorten and increase the number of soybean leaves without significantly reducing yields.

A final cause for concern is that most CFCs remain in the atmosphere for several decades, retaining their ability to destroy ozone. This is because CFCs are destroyed only after moving very slowly into the stratosphere, where they are broken up by ultraviolet radiation from the sun. Unfortunately, this is also the process that leads to ozone destruction. Thus, even if we stop adding CFCs to the atmosphere today, "good" ozone concentrations will remain depressed, and destructive $u v-b$ radiation will continue to be enhanced far into the future.

\section{Montreal Protocol}

These developments led to the drafting of the Montreal Protocol, one of the most comprehensive international agreement ever established. The protocol was developed in 1984 and amended in the London (1987) and Copenhagen (1992) Accords. It sets out a number of stipulations including:

1. an exhaustive inventory of all potential ozone-depleting and "transitional" materials;

2. specific production quotas on some of those materials;

3 . procedures for recovering and recycling current stocks of those materials;
A

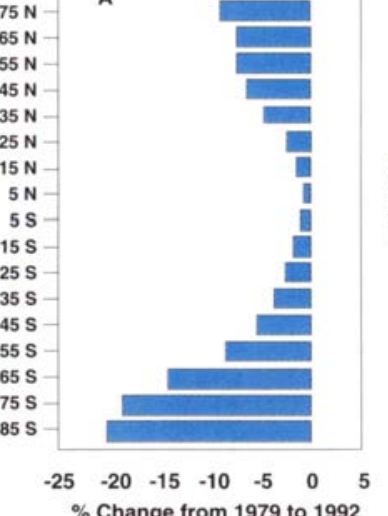

$\%$ Change from 1979 to 1992

Fig. 2. Percent of change from 1979 to 1992 for each latitudinal zone of (A) the total amount of ozone in the atmosphere as measured by satellite, and $(B)$ the calculated incidence of basal cell carcinoma based upon (A) and physiological data.

4. provisions to prohibit cheating by "non-member" countries;

5. aid to developing countries to meet its provisions;

6. requirements for the reporting of updated science;

7. provisions for regular modification and expansion of the controls;

8. regular follow-up meetings.

Remarkably, nearly all of the countries of the world, including Russia, China and India, have signed and agreed to the provisions of these agreements.

One aspect of the international agreement will affect all Americans, particularly those in agriculture. It is a provision identifying substances that will be regulated by fixed dates. Most importantly, the 1992 Copenhagen Accord specifies there will be no new production of the ubiquitous CFCs carbon tetrachloride and methyl chloroform (primarily used as industrial solvents) after December 31, 1995. The agreement also specifies that current production of CFCs should not exceed 25\% of 1986 production rates. Production of halons (bromide-containing substances used primarily in automated fire-fighting systems) was banned as of December 31, 1993. Production of other materials will be limited beginning in January. U.S. environmental laws may accelerate or expand upon certain provisions of the protocol.

\section{Impact on agriculture}

The most important effect of the Montreal Protocol on agriculture and its related industries is the ban on CFC production. The nature of these changes is outlined in table 1. Column 1 of the table lists groups of CFC uses; agriculture is heavily involved in the first four. Column 2 lists the most common CFCs used in each area. The most common materials are CFC- 11 and CFC-12 and HCFC-22 (hydrochlorofluorocarbon). "Ozone-depletion potential" and "global-warming potential," are relative to that of CFC-11, the chemical found to be most destructive of ozone. These potentials are based upon calculations using laboratory measurements of the properties of each material. The table also shows possible substitutes for materials whose production will be stopped. These substitutes include HCFCs, which release a small amount of chlorine in the stratosphere, and HFCs (hydrofluorocarbons), which release no ozone-destroying chlorine molecules.

The most common freons, CFC-11 and CFC-12, are used extensively in agriculture and related industries. Uses include nearly all industrial chillers, such as used by wineries and packers; most commercial refrigeration, such as in warehouses and supermarkets; and nearly all automobile, truck and ship refrigeration and airconditioning systems. Most home refrigerators, air-conditioning units and foam production units use HCFC-22, whose production will be phased out gradually. Beginning January 1, 1996, total HCFC production, including production of HCFC-22, will be limited to approximately the 1989 production total. Production of HCFCs then will be reduced to $35 \%$ of 1989 production in $2010,10 \%$ in $2015,0.5 \%$ in 2020 and zero in 2030.

Thus, nearly all of agriculture and related industries will be impacted by the Montreal Protocol's ban on future CFC production. Since the CFCs are only a danger when released into the atmosphere, most users will be affected by the ban only when their re- 
frigeration system leaks, needs repair or requires replacement. In some cases these impacts will be relatively slight. For those whose systems use HCFC22 , the only immediate impact will likely be an increase in its price. For other systems, when repair or replacement is required, owners will have the options of (1) utilizing dwindling and progressively costlier stocks of recycled quantities of CFCs; (2) converting systems to an available substitute; or (3) replacing systems with ones specifically designed to use a substitute.

The least expensive option in the short run will probably be to use recycled stocks. However, costs of CFC11 and CFC-12 are expected to more than double by 1996. Conversion of existing systems will often involve relatively simple changes such as a substitution of the compressor oil and/or replacement of gaskets. However, these converted systems will be 5 to $20 \%$ less efficient, increasing electrical power costs and decreasing maximum cooling capacity. Further improvements are possible, including changes in impeller size and/or speed, but could end up making the cost of conversion more expensive than replacing the system with one designed to use a substitute.

Users will also have to decide which substitute to use. An important consideration here is the fact that HCFCs will have strong production constraints early in the next century. Concern has also been expressed about the safety of some substitutes and their tendency to accelerate degradation of system components. For instance, some substitutes are so toxic they require special installation procedures, and are considered inappropriate for use near people, such as in supermarket refrigeration. Consideration also must be given to the global warming potential of substitutes, since those with high global warming potentials probably will be regulated in the future. Furthermore, less efficient systems will contribute indirectly to global warming since the ultimate sources of energy to run refrigerating systems are usually natural gas and coal, both of which emit carbon dioxide, the primary global warming gas.

Besides the consequences of the Montreal Protocol's ban on CFCs, agriculture will also be affected by new regulations on the use and production of methyl bromide (table 1). This material has a relatively short lifetime in the atmosphere (approximately 1.5 years), but per molecule is a much more effective destroyer of ozone than are CFCs. Methyl bromide is used extensively in developed countries as a soil and produce fumigant. In devel-

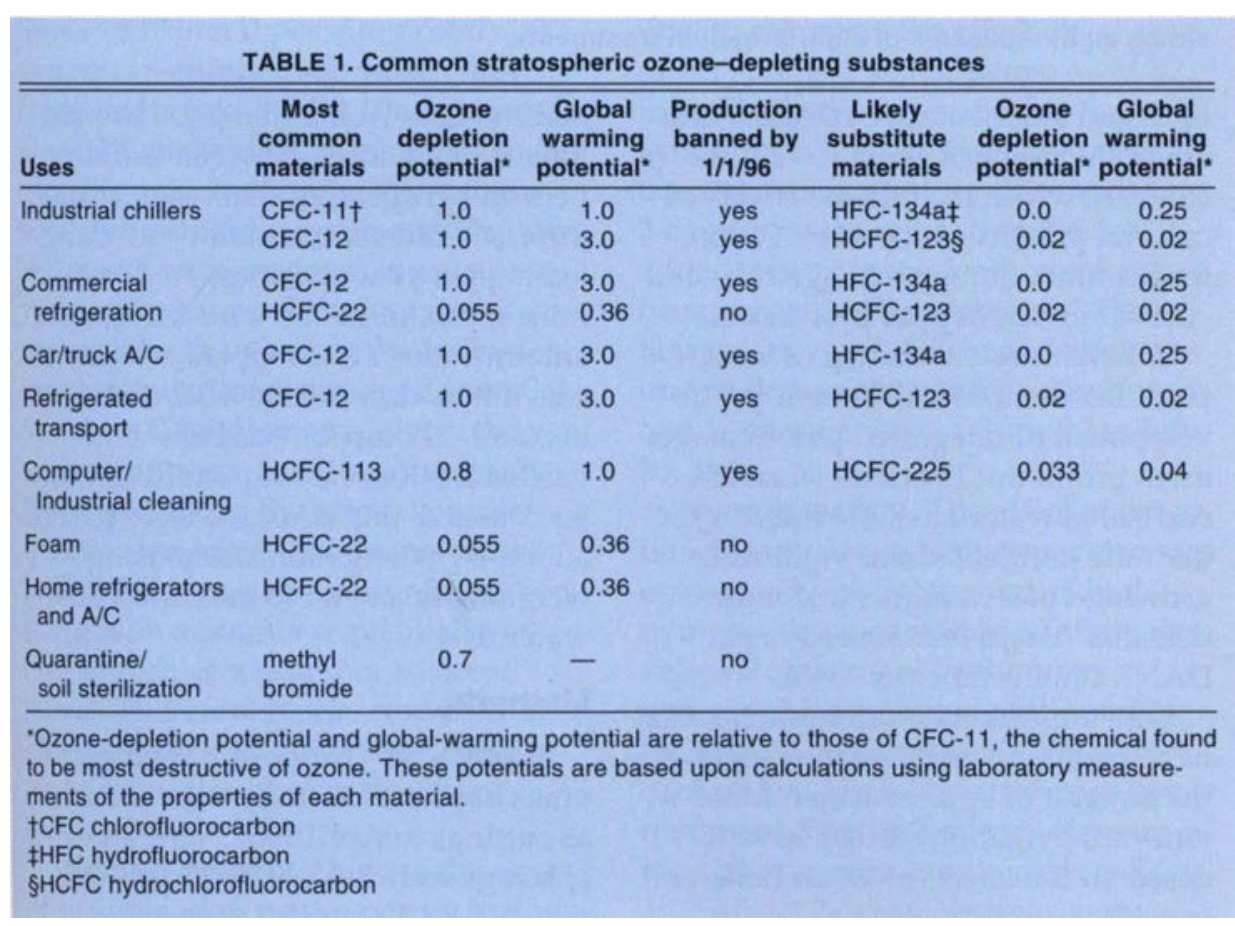

oping countries, methyl bromide is also a quarantine fumigant, required for fruits, vegetables, forestry products, cotton, cocoa, coffee beans, and grains before export to developed countries. In 1995, production of nonquarantine methyl bromide will be frozen at the 1991 level; future controls are likely.

\section{Conclusions}

This report has outlined some of the theory and observations behind the assertion that stratospheric ozone concentrations are decreasing due to human-caused releases of chlorineand bromine-containing substances, and that these decreases could have a substantial impact on human health and agricultural production. It has also outlined the international response to this problem, including limits on production of many important chemical materials used in and outside agriculture. It has tried to outline the decisions individual farmers, food processors, and others may face in response to curtailed production of these materials. However, it has not been the intent of this paper to fully explore the available options or to recommend specific actions. Frequently those will be made by refrigeration suppliers and contractors. However, it is hoped this paper will give agricultural industry owners and managers a better understanding of their options so they can make the best long-range decisions for their businesses and the environment.

B.C. Weare is Professor, Atmospheric Science Program, UC Davis. This work was supported by the UC Agricultural Experiment Station.

The author thanks the editors of the Global Environmental Change Report, published by the Cutter Information Corporation, and all of the students and colleagues who have helped him better understand the wide range of subjects discussed in this paper. Figure 1 is largely based upon the 1989 review articles in American Scientist by Rowland and in Scientific American by Graedel and Crutzen. Data in figure 2 were taken from Madronich and Gruijl, Science, 366, p. 23 (1993). Table 1 is based primarily upon reports provided by Dr. Mac McFarland of the Dupont Corporation. 